\title{
Stochastic Analysis of Network Coding Based Relay-Assisted I2V Communications in Intelligent Transportation Systems
}

\author{
Weijun Xing, ${ }^{1}$ Fuqiang Liu, ${ }^{1}$ Chao Wang, ${ }^{1,2}$ and Ping Wang ${ }^{1}$ \\ ${ }^{1}$ School of Electronics and Information Engineering, Tongji University, Shanghai, China \\ ${ }^{2}$ Key Laboratory of System Control and Information Processing, Ministry of Education, Shanghai, China \\ Correspondence should be addressed to Chao Wang; chaowang@tongji.edu.cn
}

Received 26 May 2017; Revised 25 July 2017; Accepted 2 August 2017; Published 24 September 2017

Academic Editor: Ke Guan

Copyright (C) 2017 Weijun Xing et al. This is an open access article distributed under the Creative Commons Attribution License, which permits unrestricted use, distribution, and reproduction in any medium, provided the original work is properly cited.

\begin{abstract}
We investigate the information transmission in a typical communication scenario in the intelligent transportation systems (ITS), that is, infrastructures providing ITS services that intend to broadcast independent messages to the vehicles within their coverage areas. Due to the dynamic nature of wireless signal propagation links and interuser interference, it is hard to guarantee satisfactory performance by direct infrastructure to vehicle (I2V) transmissions. To solve this problem, we propose allowing certain vehicles to serve as relays to assist in the information distribution process and applying a class of finite-field network codes to efficiently use the available spectrum resources. Considering that the infrastructure information sources and all vehicles are randomly distributed following Poisson point processes, we model a general urban ITS communication network and based on the stochastic geometry we derive the probability that a vehicle can successfully recover all the desired messages from its serving infrastructure. The analytical and numerical results clearly demonstrate that our proposed network coding based relay-assisted I2V transmission can significantly improve the communication performance of the conventional direct $\mathrm{I} 2 \mathrm{~V}$ transmission strategy.
\end{abstract}

\section{Introduction}

With the rapid increase of the number of vehicles driving on the road, nowadays, traffic accidents and congestion become severe global problems and thus draw concerns all over the world. To handle this issue, the concept of vehicular ad hoc networks (VANETs) has been proposed to serve as an effective solution. In VANETs, vehicles' status information (e.g., real-time position, velocity, and driving direction) can be periodically shared with others in the vicinity through wireless communication technologies to provide a detailed environment awareness to drivers. Early warning messages can also be broadcast when an emergency situation occurs to reduce drivers' reaction time. Due to the high mobility and limited wireless data transmission range of vehicles, to improve the network connectivity and coverage, roadside infrastructures are considered to be deployed along the roads to relay vehicles' messages and distribute other traffic-related information (e.g., traffic lights' signals, road congestion status, and regulatory speed limits [1]). These approaches potentially bring significant improvements to traffic safety and transportation efficiency [1-3].

Because of the aforementioned advantages, VANETs have attracted a large amount of research attentions. The IEEE 802.11p standard based on the Dedicated Short-Range Communication (DSRC) technology has been developed to support intelligent transportation systems (ITS) applications in VAENTs since 2004 [2]. Due to the lack of pervasive infrastructures deployment and sufficient transmission range, the DSRC technology is normally considered to offer intermittent and short-lived connectivity between roadside infrastructures and vehicles [4]. Recently, the third Generation Partnership Project (3GPP) has considered vehicular communications as an important application scenario of the fifth generation (5G) cellular systems [5]. It is envisioned that, in 5G systems, several types of infrastructures, potentially including eNodeBs, eNodeB-type roadside units (RSUs), and UE-type RSUs, can be deployed to provide ITS services to vehicles within their coverage areas [5]. Clearly, the overall connectivity of vehicular communication networks can be significantly improved through the infrastructure to vehicle 
(I2V) data transmissions. However, since the available spectrum resources allocated to vehicular networks are in general limited, having a large number of infrastructures may also generate severe cochannel interference issues and hence affect the quality of service (QoS) in practice.

The analysis of $\mathrm{I} 2 \mathrm{~V}$ transmission strategies has been investigated by several recent research works. It has been shown that properly designed transmission schemes can lead to significant performance improvement in terms of enhanced capacity [3] and energy efficiency $[6,7]$ and reduced delay $[8,9]$. However, in most current works, all roadside infrastructures and vehicles are considered to be located along a single road. Hence each vehicle's desired signals and interference signals are modeled to come from a onedimensional space. Clearly this is hardly the case in practice, since normally there are multiple roads within a certain area and the interference signals may come from all directions. The deployment of multiple roads is taken into account in [10]. But all infrastructures are still assumed to locate only along each road, which leads to the one-dimensional homogeneous Poisson point processes (H-PPPs) model. It is expected that multiple types of infrastructures (e.g., eNodeBs) can serve to provide $\mathrm{I} 2 \mathrm{~V}$ communication applications. Hence this model may still be unable to reflect the most general conditions in future ITS, where some cochannel interference generators may randomly distribute in the $2 \mathrm{D}$ space [11].

To further improve the performance of $\mathrm{I} 2 \mathrm{~V}$ communications, [11-14] propose using extra relay terminals (e.g., vehicles running on the roads) to assist in the message delivery procedure. Requiring relays to repeat the infrastructure messages would provide vehicle receivers with spatial diversity gain that can combat the negative impact of the complex fading and interference characteristics in vehicular communication environments. Although clear performance enhancement can be observed, the repetition coding operation adopted at relays may in fact still be spectrally inefficient [15]. This is because that each relay uses multiple orthogonal channels to separately repeat the source's messages, which potentially requires an unnecessarily large channel usage. It is well known that for this issue the concept of network coding [16] serves as an elegant solution. Instead of individually retransmitting the source messages, each relay combines them to form a new codeword such that a single channel usage suffices to deliver it to the intended destination. To realize efficient transmission of multiple independent messages, a class of maximum distance separable finite-field network codes (MDS-FFNCs), whose coding principle lies in the summation in higher order finite fields, is hence proposed by [17].

In this paper, we propose applying MDS-FFNC to relayassisted I2V communications in ITS. The system model considers the wireless distribution of multiple independent messages from roadside infrastructures to the vehicles in their coverage areas. Some vehicles can be selected to act as relays and apply MDS-FFNC to assist in the $12 \mathrm{~V}$ transmissions in order to improve system performance. Based on stochastic geometry, the distribution of vehicles and roadside infrastructures are modeled following two independent PPPs. We derive the successful transmission probability in such a complex system. Through comparisons with the classic direct $\mathrm{I} 2 \mathrm{~V}$ transmissions, the potential benefits of exploiting relay and network coding techniques in I2V communication systems are exhibited.

The remainder of this paper is organized as follows. We first present the system model and our proposed transmission scheme in Section 2. The successful transmission probability analysis is introduced in Section 3. Section 4 demonstrates comparisons between our proposed scheme and two benchmark schemes via numerical results. Finally, Section 5 concludes the paper.

\section{System Model}

In this section, we first introduce the system model and then present the proposed network coding based relay-assisted $\mathrm{I} 2 \mathrm{~V}$ transmission process.

2.1. System Model. We consider an urban road system in the infinite plane $\mathbb{R}^{2}$ with a lattice pattern as shown in Figure 1(a). The system consists of many horizontal roads separated by distance $d_{\text {road, } X}$ meters (denoted by $X_{i}, i \in \mathbb{Z}$ and $\mathbb{Z} \triangleq\{\ldots,-1,0,1, \ldots\})$ and many vertical roads separated by distance $d_{\text {road, } Y}$ meters (denoted by $Y_{j}, j \in \mathbb{Z}$ ). It is common to see this kind of road system in many cities, such as New York and Barcelona [18]. The locations of the vehicles running on these roads are considered to be distributed following onedimensional H-PPPs [10]. The wireless transceiver module equipped at each vehicle is activated with a certain probability to broadcast the vehicle's status information to surrounding vehicles. At any time instant, the simultaneously activated vehicles running on the horizontal road $X_{i}$, denoted by a set $\Pi_{X_{i}}$, can thus be modeled by a PPP with density $\lambda_{X_{i}}$. Similarly, the simultaneously activated vehicles running on the vertical road $Y_{j}$, denoted by set $\Pi_{Y_{j}}$, are modeled by a PPP with density $\lambda_{Y_{j}}$. The ITS infrastructures are assumed to be randomly deployed in the plane $\mathbb{R}^{2}$. Each infrastructure is also activated with a probability to broadcast traffic status information to the vehicles running on a road (these vehicles would normally be interested in the same information) within its coverage area. At each time instant, the locations of the simultaneously activated infrastructures, denoted by a set $\Pi_{S}$, can be modeled to follow an independent twodimensional PPP with intensity $\lambda_{S}[11]$.

Let us consider the $\mathrm{I} 2 \mathrm{~V}$ information transmission from one of the ITS infrastructures to one of the vehicles within its coverage area. Denote the infrastructure by $S$ and the target vehicle by $D$. Without loss of generality, we assume that $D$ is running on the road $X_{0}$. S intends to send a set of $M \geq 1$ independent messages $\mathscr{I}=\left\{I_{S_{1}}, \ldots, I_{S_{M}}\right\}$, which may contain rich information such as traffic lights' signals, road congestion status, and speed limits, to $D$. Due to the other simultaneously activated infrastructures and vehicles, the reception of $D$ would be corrupted by cochannel interference so that a satisfactory quality of message delivery may not be attainable.

To handle this issue, we adopt the concept of cooperative communications and select a vehicle $R$ also running on $X_{0}$ to serve as a relay to help $D$ through a vehicle-to-vehicle (V2V) 


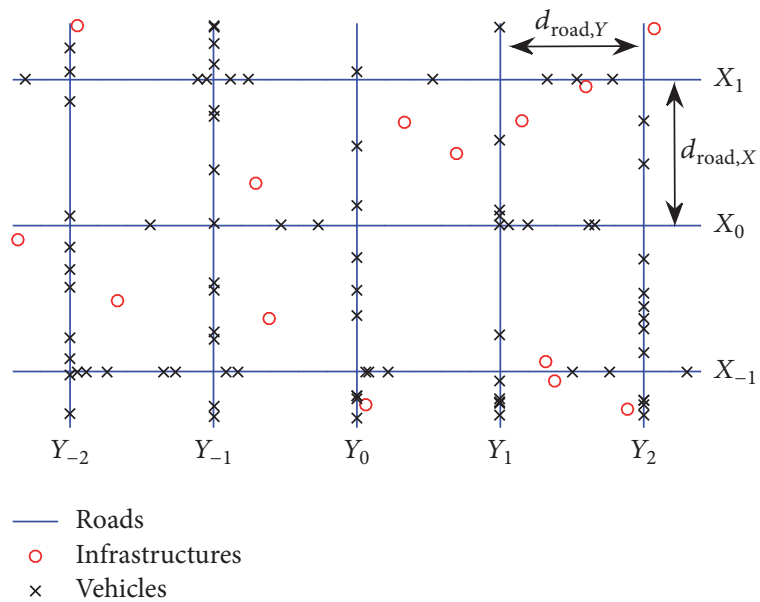

(a) An urban road system

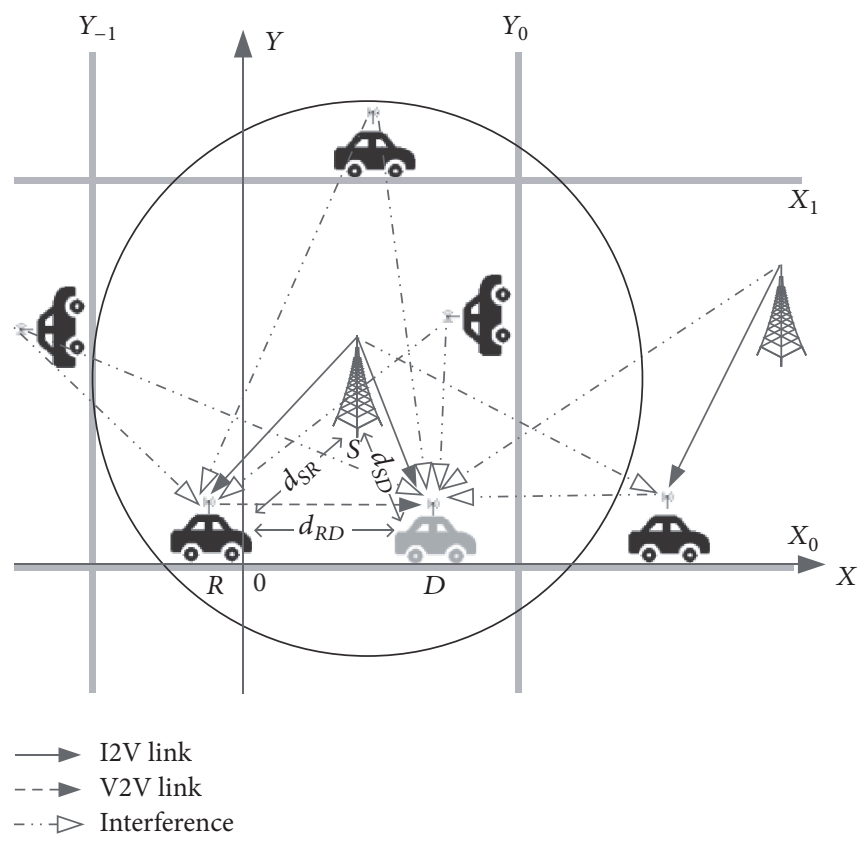

(b) An example relay-assisted I2V communication network

FIGURE 1: Scenario of network coding based relay-assisted I2V communications.

communication link (actually $R$ can be selected to help all vehicles on $X_{0}$ who are interested in $\mathscr{I}$ ). Due to hardware limitation, $R$ 's relay function is operated in a half-duplex decodeand-forward (DF) fashion. This example of relay-assisted I2V communication network is illustrated in Figure 1(b).

The message transmissions are carried out in a slow, frequency-flat Rayleigh block fading environment. One message is transmitted using one unit time slot. The small-scale fading coefficient between transmitter $a$ and receiver $b$ is denoted as $h_{a b}$ and is modeled as a complex Gaussian random variable with zero mean and unit variance. $h_{a b}$ remains constant within each time slot and changes independently afterwards. The impact of large-scale fading on signal power is modeled by $d_{a b}^{-\alpha_{a b}}$, in which $d_{a b}$ is the distance between $a$ and $b$ and $\alpha_{a b}$ is the path loss exponent. By this means, at each time slot, the relationship between the transmit power at node $a$ and receive power at node $b$ can be expressed as the following form:

$$
P_{b}=\left|h_{a b}\right|^{2} d_{a b}^{-\alpha_{a b}} P_{a} .
$$

For presentation simplicity, we set the transmit power of all infrastructures to be $P_{S}$ and that of vehicles to be $P_{V}$. The value of $\alpha_{a b}$ can be very different for different transmitter-receiver pairs, especially when both line-of-sight and non-line-ofsight signal propagations are taken into consideration. To allow the performance analysis in the considered complex system to be mathematically tractable, in this paper, we set this value to be the same as $\alpha_{I}$ for all interference links. Compared with the I2V links from $S$ to $R$ and $S$ to $D$, the path loss exponent for the V2V R-D link may be smaller due to the relative shorter distance and simpler fading characteristics between them. For instance, following [18, 19], we can choose $\alpha_{S R}=\alpha_{S D}=4$ and $\alpha_{R D}=2$. For other choices of the path loss exponents, the analytical method provided in this paper can also be applied.

Therefore, at each time slot, the received signal-tointerference-plus-noise ratio (SINR) between transmitter $a$ $(a \in\{S, R\})$ and receiver $b(b \in\{R, D\})$ can be expressed as the following form:

$$
\operatorname{SINR}_{a b}=\frac{P_{a}\left|h_{a b}\right|^{2} d_{a b}^{-\alpha_{a b}}}{Z_{S}+Z_{X}+Z_{Y}+\sigma^{2}},
$$

where $Z_{S}=\sum_{S_{k} \in \Pi_{S}}\left|h_{S_{k} b}\right|^{2} d_{S_{k} b}^{-\alpha_{I}} P_{S}, Z_{X}=\sum_{i \in \mathbb{Z}} Z_{X_{i}}=$ $\sum_{i \in \mathbb{Z}} \sum_{x \in \Pi_{X_{i}}}\left|h_{x b}\right|^{2} d_{x b}^{-\alpha_{I}} P_{V}, \quad Z_{Y}=\sum_{j \in \mathbb{Z}} Z_{Y_{j}}=$ $\sum_{j \in \mathbb{Z}} \sum_{y \in \Pi_{Y_{j}}}\left|h_{y b}\right|^{2} d_{y b}^{-\alpha_{I}} P_{V}$ denote the received interference power from infrastructures in $\Pi_{S}$ and vehicles in $\Pi_{X_{i}}$ and $\Pi_{Y_{j}}$ for all $i, j \in \mathbb{Z}$, respectively, and $\sigma^{2}$ denotes noise power.

2.2. Transmission Process. In this paper, we require the relay to combine the $M$ source messages $I_{S_{1}}, \ldots, I_{S_{M}}$ into $L$ new messages $I_{R_{1}}, \ldots, I_{R_{L}}$ using the MDS-FFNC. Specifically, define vectors $\mathbf{I}_{\mathbf{S}}=\left[I_{S_{1}}, \ldots, I_{S_{M}}\right]^{T}$ and $\mathbf{I}_{\mathbf{R}}=\left[I_{R_{1}}, \ldots, I_{R_{L}}\right]^{T}$, where $[\cdot]^{T}$ is the vector transpose operator. The input-output relation of the network coding procedure can be expressed as

$$
\left[\begin{array}{c}
\mathbf{I}_{\mathbf{S}} \\
\mathbf{I}_{\mathbf{R}}
\end{array}\right]=\underbrace{\left[\begin{array}{cccc}
1 & 0 & \cdots & 0 \\
\vdots & \vdots & \ddots & \vdots \\
0 & 0 & \cdots & 1 \\
\omega_{R_{1}, S_{1}} & \omega_{R_{1}, S_{2}} & \cdots & \omega_{R_{1}, S_{M}} \\
\vdots & \vdots & \ddots & \vdots \\
\omega_{R_{L}, S_{1}} & \omega_{R_{L}, S_{2}} & \cdots & \omega_{R_{L}, S_{M}}
\end{array}\right]}_{G}
$$




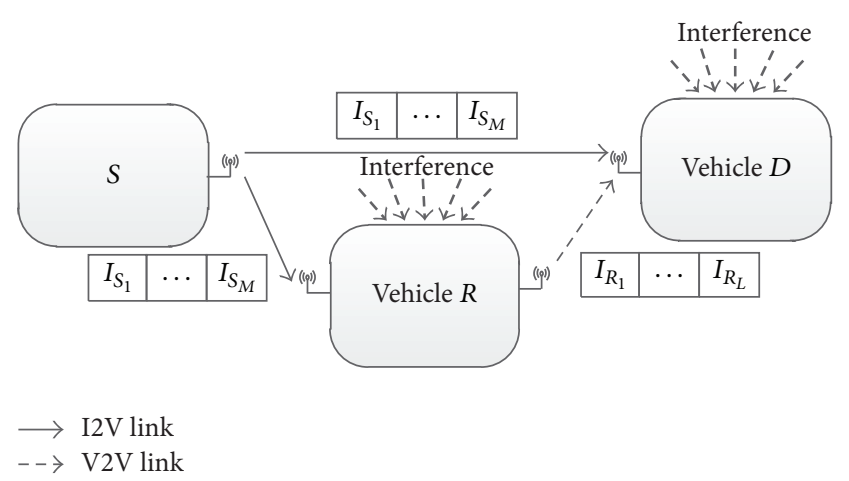

FIGURE 2: Transmission process of the proposed NC scheme.

where all summations are carried out in a certain finite field, and the coding coefficients $\omega_{R_{j}, S_{i}}$ are designed to guarantee that the global encoding kernels (GEK) [17] are linearly independent. By this means, a submatrix constituted by any $M$ rows of the transfer matrix $G$ is nonsingular. Any $M$ out of the $M+L$ codewords expressed on the left-hand side of (3) are sufficient to help recover the whole source message set $\mathscr{I}$.

The transmission process in the considered relay-assisted $\mathrm{I} 2 \mathrm{~V}$ communication system is shown in Figure 2 and is termed network-coded cooperation (NC). A total of $M+L$ time slots are used to complete the transmission of $\mathscr{I}$ from $S$ to $D$. The first $M$ slots are assigned to $S$ to broadcast $I_{S_{1}}, \ldots, I_{S_{M}}$, respectively. If the relay $R$ can decode all the $M$ messages, in the next $L$ time slots it transmits the network-coded messages $I_{R_{1}}, \ldots, I_{R_{L}}$. Otherwise, $R$ remains silent. After $S$ and $R$ complete their transmissions, $D$ tries to recover $\mathscr{I}$ based on its received signals. Note that the choice of the value $L$ can affect the system performance. Having a large value of $L$ would provide high diversity to the I2V transmission process. But since a large amount of channel usage is required, the spectral efficiency would be reduced as a potential cost. When $L$ is chosen to be zero, the relay is not used to assist in the intended I2V communications. This is termed direct transmission (DT) throughout the paper.

To evaluate the performance of the proposed NC scheme, we will derive the probability that $D$ can successfully recover all $M$ source messages. This task will be carried out in the following section.

\section{Performance Analysis}

As described in Section 2.2, in the transmission process of the NC scheme, there are two stages to conduct message delivery, that is, $S$ first broadcasts $M$ messages to $D$ and $R$ via I2V links and then $R$ transmits $L$ network-coded messages to $D$ via the V2V link. The final probability that $D$ can successfully recover the source message set $\mathscr{I}$ relies on the successful transmission probability at each time slot. To this end, we first derive the latter probability expression.

We have seen that, at each time slot, the SINR between transmitter $a(a \in\{S, R\})$ and receiver $b(b \in\{R, D\})$ can be expressed by $\operatorname{SINR}_{a b}$ as shown in (2). If $\operatorname{SINR}_{a b}$ is larger than a certain threshold $T$, the receiver $b$ can correctly decode its received signal from $a$. Hence the successful transmission probability, denoted by $P_{\text {suc }, a b}$, can be expressed as $P_{\text {suc }, a b}=$ $\operatorname{Pr}\left\{\operatorname{SINR}_{a b} \geq T\right\} . P_{\text {suc }, a b}$ can be further derived as (4), where $\mathscr{L}_{Z_{S}}(\cdot), \mathscr{L}_{Z_{X}}(\cdot)$, and $\mathscr{L}_{Z_{Y}}(\cdot)$ are the Laplace transform of $Z_{S}$, $Z_{X}$, and $Z_{Y}$, respectively. In fact, $\mathscr{L}_{Z_{\mathrm{S}}}(\cdot), \mathscr{L}_{Z_{X}}(\cdot)$, and $\mathscr{L}_{Z_{Y}}(\cdot)$ represent the reduction of successful decoding probability due to the cochannel interference generated by interfering infrastructures, vehicles running on the horizontal roads, and vertical roads, respectively. In what follows, we derive the closed-form expressions of these three factors.

$$
\begin{aligned}
& P_{\text {suc }, a b}=\operatorname{Pr}\left\{\frac{P_{a}\left|h_{a b}\right|^{2} d_{a b}^{-\alpha_{a b}}}{Z_{S}+Z_{X}+Z_{Y}+\sigma^{2}} \geq T\right\} \\
& =\mathbb{E}_{Z_{S}, Z_{X}, Z_{Y}}\left[\operatorname { P r } \left\{\left|h_{a b}\right|^{2}\right.\right. \\
& \left.\left.\geq \frac{T d_{a b}^{\alpha_{a b}}}{P_{a}}\left(Z_{S}+Z_{X}+Z_{Y}+\sigma^{2}\right)\right\}\right] \\
& =\mathbb{E}_{Z_{S}, Z_{X}, Z_{Y}}\left[\exp \left(-\frac{T d_{a b}^{\alpha_{a b}}}{P_{a}} Z_{S}\right) \exp \left(-\frac{T d_{a b}^{\alpha_{a b}}}{P_{a}} Z_{X}\right)\right. \\
& \left.\cdot \exp \left(-\frac{T d_{a b}^{\alpha_{a b}}}{P_{a}} Z_{Y}\right)\right] \exp \left(-\frac{T d_{a b}^{\alpha_{a b}} \sigma^{2}}{P_{a}}\right) \\
& =\mathscr{L}_{Z_{S}}\left(\frac{T d_{a b}^{\alpha_{a b}}}{P_{a}}\right) \mathscr{L}_{Z_{X}}\left(\frac{T d_{a b}^{\alpha_{a b}}}{P_{a}}\right) \mathscr{L}_{Z_{Y}}\left(\frac{T d_{a b}^{\alpha_{a b}}}{P_{a}}\right) \\
& \cdot \exp \left(-\frac{T d_{a b}^{\alpha_{a b}} \sigma^{2}}{P_{a}}\right) .
\end{aligned}
$$

Let $s=T d_{a b}^{\alpha_{a b}} / P_{a}$. Since the locations of the ITS infrastructures are assumed to be randomly distributed following a two-dimensional PPP, the Laplace transform of the aggregate interference from simultaneously activated infrastructures is given by

$$
\mathscr{L}_{Z_{S}}(s)=e^{-\lambda_{S} \pi\left(s P_{S} / P_{a}\right)^{2 / \alpha_{I}} \Gamma\left(1+2 / \alpha_{I}\right) \Gamma\left(1-2 / \alpha_{I}\right)},
$$

where $\Gamma(z)=\int_{0}^{\infty} e^{-u} u^{z-1} d u$ is the gamma function [20].

The locations of all vehicles are distributed according to one-dimensional H-PPPs and vehicles on different roads are independent. The Laplace transform of the aggregated interference from interfering vehicles $\mathscr{L}_{Z_{X}}(s)$ and $\mathscr{L}_{Z_{Y}}(s)$ can be expressed as

$$
\begin{aligned}
& \mathscr{L}_{Z_{X}}(s)=\prod_{i \in \mathbb{Z}} \mathscr{L}_{Z_{X_{i}}}(s), \\
& \mathscr{L}_{Z_{Y}}(s)=\prod_{j \in \mathbb{Z}} \mathscr{L}_{Z_{Y_{j}}}(s) .
\end{aligned}
$$

To obtain the expressions of $\mathscr{L}_{Z_{X}}(s)$ and $\mathscr{L}_{Z_{Y}}(s)$, we should first derive $\mathscr{L}_{Z_{X_{i}}}(s)$ and $\mathscr{L}_{Z_{Y_{j}}}(s)$. We can see that $Z_{X_{i}}$ and $Z_{Y_{j}}$ are the aggregated interference received from the vehicles on the roads $X_{i}$ and $Y_{j}$. Without loss of generality, 
we set the coordinates of the location of the receiver $b$ to be $(0,0)$ in $\mathbb{R}^{2}$ (road $X_{0}$ is the horizontal axis) and the location of the intersection of $X_{0}$ and $Y_{0}$ is set to be $\left(d_{b I}, 0\right)$.

Let the location coordinate of the $v$ th interfering vehicle, denoted as $x_{i, v},\left(x_{i, v} \in \Pi_{X_{i}}, v \in\{1,2,3, \ldots\}\right)$, on $\operatorname{road} X_{i}$ be $\left(x_{v}^{(i)}, y_{\text {road }}^{(i)}\right)$, where $y_{\text {road }}^{(i)}=i \cdot d_{\text {road, } X}$, and that of the $q$ th interfering vehicle, denoted as $y_{j, q}\left(y_{j, q} \in \Pi_{Y_{j}}, q \in\{1,2\right.$, $3, \ldots\})$, on road $Y_{j}$ be $\left(x_{\text {road }}^{(j)}, y_{q}^{(j)}\right)$, where $x_{\text {road }}^{(j)}=j \cdot d_{\text {road, } Y}$. The distance between $x_{i, v}$ and the receiver $b$ is $d_{x_{i, v} b}=$ $\sqrt{\left(x_{v}^{(i)}\right)^{2}+\left(y_{\text {road }}^{(i)}\right)^{2}}$ and we have $\left(d / d x_{v}^{(i)}\right) d_{x_{i, v} b}=x_{v}^{(i)} / d_{x_{i, v} b}$. The Laplace transform $\mathscr{L}_{Z_{X_{i}}}(s)$ is given by

$$
\begin{aligned}
\mathscr{L}_{Z_{X_{i}}}(s) & =\mathbb{E}\left[\exp \left(-s Z_{X_{i}}\right)\right]=\mathbb{E}\left[\prod_{x_{i, v} \in \Pi_{X_{i}}} \exp \left(-s P_{v}\left|h_{x_{i, v},}\right|^{2} d_{x_{i, v} b}^{-\alpha_{I}}\right)\right] \stackrel{(a)}{=} \mathbb{E}_{\Pi_{X_{i}}}\left[\prod_{x_{i, v} \in \Pi_{X_{i}}} \mathbb{E}_{h_{x_{i, v}}}\left\{\exp \left(-s P_{V}\left|h_{x_{i, v} b}\right|^{2} d_{x_{i, v} b}^{-\alpha_{I}}\right)\right\}\right] \\
& \stackrel{(b)}{=} \mathbb{E}_{\Pi_{X_{i}}}\left[\prod_{x_{i, v} \in \Pi_{X_{i}}} \frac{1}{1+\left(1 / s P_{V}\right) d_{x_{i, v} b}^{\alpha_{I}}}\right] \stackrel{(c)}{=} \exp \left(-\int_{-\infty}^{+\infty} \frac{\lambda_{X_{i}}}{1+\left(1 / s P_{V}\right)\left(\sqrt{\left(x_{v}^{(i)}\right)^{2}+\left(i \cdot d_{\text {road, }}\right)^{2}}\right)^{\alpha_{I}}} d x_{v}^{(i)}\right) \\
& =\exp \left(-2 \lambda_{X_{i}} \int_{y_{\text {road }}^{(i)}}^{+\infty} \frac{d_{x_{i, v} b} d d_{x_{i, v} b}}{\left.\sqrt{\left(d_{x_{i, v} b}\right)^{2}-\left(y _ { \text { road } } ^ { ( i ) } \left(1+\left(1 / s P_{V}\right) d_{x_{i, v} b}^{\alpha_{I}}\right.\right.}\right)}\right) \\
& =\exp \left(-\lambda_{X_{i}}\left(s P_{V}\right)^{1 / \alpha_{I}} \int_{\omega_{0}}^{+\infty} \frac{1}{\sqrt{\omega-\omega_{0}}\left(1+\omega^{\alpha_{I} / 2}\right)} d \omega\right)
\end{aligned}
$$

where (a) follows from the independence of the fading parameters, $(b)$ follows from the Laplace transform of the Rayleigh fading (i.e., $\mathscr{L}(s)=1 /(1+s)$ ), (c) follows from the expression of the probability generating function for a PPP [21], $\omega=\left(d_{x_{i, b} b} /\left(s P_{V}\right)^{1 / \alpha_{I}}\right)^{2}$, and $\omega_{0}=\left(y_{\text {road }}^{(i)} /\left(s P_{V}\right)^{1 / \alpha_{I}}\right)^{2}$. As we mentioned earlier, the path loss exponent $\alpha_{I}$ can be chosen according to the applied signal propagation environments. The expression of (8) can be derived for each individual choice of $\alpha_{I}$. For instance, setting $\alpha_{I}=4$ provides us with the following integral:

$$
\int_{\omega_{0}}^{+\infty} \frac{1}{\sqrt{\omega-\omega_{0}}\left(1+\omega^{\alpha_{I} / 2}\right)} d \omega
$$

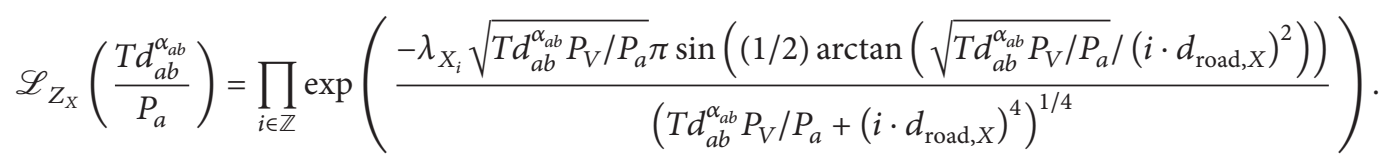

Similarly, when $\alpha_{I}=4$, the expression of $\mathscr{L}_{Z_{Y_{j}}}(s)$ can be derived as

$$
\mathscr{L}_{Z_{Y_{j}}}(s)=\exp \left(\frac{-\lambda_{Y_{j}} \sqrt{s P_{V}} \pi \sin \left((1 / 2) \arctan \left(\sqrt{s P_{V}} /\left(d_{b I}+x_{\text {road }}^{(j)}\right)^{2}\right)\right.}{\left(s P_{V}+\left(d_{b I}+x_{\text {road }}^{(j)}\right)^{4}\right)^{1 / 4}}\right)
$$


Substituting (12) and $s=T d_{a b}^{\alpha_{a b}} / P_{a}$ into (7) leads to the Laplace transform of the aggregate interference from simultaneously activated vehicles on the horizontal roads as (13). Substitute (5), (11), and (13) into (4). We can obtain the closed-form expression of the successful transmission probability between the transmitter $a(a \in\{S, R\})$ and receiver $b(b \in\{R, D\})$ at each individual time slot, that is, $P_{\text {suc, } a b}$.

$$
\mathscr{L}_{Z_{Y}}\left(\frac{T d_{a b}^{\alpha_{a b}}}{P_{a}}\right)=\prod_{j \in \mathbb{Z}} \exp \left(\frac{-\lambda_{Y_{j}} \sqrt{T d_{a b}^{\alpha_{a b}} P_{V} / P_{a}} \pi \sin \left((1 / 2) \arctan \left(\sqrt{T d_{a b}^{\alpha_{a b}} P_{V} / P_{a}} /\left(d_{b I}+j \cdot d_{\text {road }, Y}\right)^{2}\right)\right)}{\left(T d_{a b}^{\alpha_{a b}} P_{V} / P_{a}+\left(d_{b I}+j \cdot d_{\text {road }, Y}\right)^{4}\right)^{1 / 4}}\right) .
$$

Now we are ready to derive the probability that $D$ can fully recover all source messages. Denote this probability by $P_{\mathrm{NC}}$. Based on transmission process described in the Section 2.2, we can express $P_{\mathrm{NC}}$ as

$$
P_{\mathrm{NC}}=P_{\mathrm{act}} P_{\mathrm{SRD}}+\left(1-P_{\mathrm{act}}\right)\left(P_{\mathrm{suc}, S D}\right)^{M},
$$

where $P_{\text {act }}$ denotes the probability that the relay $R$ is activated, and $P_{S R D}$ is the probability that $D$ can recover $\mathscr{I}$ under the condition that the relay is activated. Clearly, the relay $R$ is activated only when it can recover all the $M$ source messages from $S$. Thus we can calculate $P_{\text {act }}$ by

$$
P_{\text {act }}=\left(P_{\text {suc }, S R}\right)^{M} \text {. }
$$

If the relay $R$ is activated, $D$ receives a total of $M+L$ messages transmitted from $S$ and $R$. Benefitting from the MDS-FFNC, as long as $M$ out of the $M+L$ messages can be decoded, all the messages $I_{S_{1}}, \ldots, I_{S_{M}}$ can be recovered. In other words, the I2V transmission of $\mathscr{I}$ is successful if at most $L$ messages are decoded with errors at $D$. Consequently, the probability of successfully recovering all the source messages at $D$ with the help of $R$ is calculated as

$$
\begin{aligned}
P_{S R D}= & \sum_{g=0}^{\min \{M, L\}}\left(\begin{array}{c}
M \\
g
\end{array}\right)\left(1-P_{\mathrm{suc}, S D}\right)^{g}\left(P_{\mathrm{suc}, S D}\right)^{M-g} \\
& \cdot\left(\sum_{w=0}^{L-g}\left(\begin{array}{c}
L \\
w
\end{array}\right)\left(1-P_{\mathrm{suc}, R D}\right)^{w}\left(P_{\mathrm{suc}, R D}\right)^{L-w}\right),
\end{aligned}
$$

where $\left(\begin{array}{c}M \\ g\end{array}\right)\left(1-P_{\text {suc }, S D}\right)^{g}\left(P_{\text {suc }, S D}\right)^{M-g}$ is the probability that $D$ cannot directly decode $g$ messages from $S$ and $\sum_{w=0}^{L-g}\left(\begin{array}{c}L \\ w\end{array}\right)(1-$ $\left.P_{\text {suc }, R D}\right)^{w}\left(P_{\text {suc }, R D}\right)^{L-w}$ is the probability that $D$ cannot decode at most $L-g$ network-coded messages from $R$.

On the other hand, if the relay cannot successfully decode all the $M$ messages from $S$, the probability of which is $1-$ $P_{\text {act }}$, then the I2V transmission from $S$ to $D$ can be successful only when $D$ can recover the whole message set $\mathscr{I}$ from $S$. The successful transmission probability in this case is clearly $\left(P_{\text {suc }, S D}\right)^{M}$. Finally, substitute (15) and (16) into (14). We can obtain the exact successful transmission probability achieved by the NC scheme.

If the relay is not used to assist the desired I2V transmission, the successful transmission probability from $S$ to $D$ is denoted by $P_{\mathrm{DT}}$ and it is certainly derived as follows using (4):

$$
P_{\mathrm{DT}}=\left(P_{\mathrm{suc}, S D}\right)^{M}
$$

\section{Numerical Results}

In this section, we evaluate the performance of the proposed network coding based relay-assisted I2V communications. Two benchmark schemes are considered to demonstrate the benefits of the proposed NC scheme. In addition to the DT scheme without using relays (i.e., direct $\mathrm{I} 2 \mathrm{~V}$ transmissions), another forwarding strategy that is commonly adopted in relay-aided $\mathrm{I} 2 \mathrm{~V}$ communication systems is to require the relay to repeat the messages generated by the infrastructure (e.g., see $[12,13])$. We term this repetition-coded cooperation (RC) scheme. This scheme is simple to realize. But when the infrastructure intends to deliver multiple messages to its serving vehicles, it may not be satisfactorily good. For instance, consider our system in which the source intends to broadcast $M$ messages. Allowing the relay to repeat each source message once would require the whole transmission to be completed using $2 M$ time slots. Each message arrives in $D$ through two independent paths, one directly from $S$ and one through $R$. Compared with our NC scheme with $L<M$, its diversity and multiplexing gains may both be insufficient. Since the calculation of the successful transmission probability of the RC scheme is involved (due to the aforementioned interference issues), we will show its performance via simulations.

For fair comparisons, we demand the average transmission data rate from the infrastructure to be the same for all three schemes. This rate is denoted by $R_{0}$ bits per message per (time) channel use (BPMPCU). The DT scheme demands $M$ time slots to complete the direct $\mathrm{I} 2 \mathrm{~V}$ transmission of $M$ messages. Hence each message should contain $M R_{0}$ bits of information. The RC scheme needs $2 M$ slots and each message has $2 M R_{0}$ bits of information. Finally, the NC scheme uses $M+L$ time slots to complete the transmission. Each message should contain $(M+L) R_{0}$ bits of information to maintain the average rate $R_{0}$ BPMPCU. Furthermore, we assume that the infrastructure source applies capacity-achieving Gaussian codes to encode its information so that the SINR thresholds of the DT, RC, and NC schemes can be expressed as $T=2^{M R_{0}}-1$, $T=2^{2 M R_{0}}-1$, and $T=2^{(M+L) R_{0}}-1$, respectively.

In the following numerical results, we use an example network topology to illustrate the performance gains obtained by the NC scheme. Specifically, we set $d_{S D}=d_{S R}=100 \mathrm{~m}$ and $d_{S D}=60 \mathrm{~m}$. In addition, the distance from $D$ to $Y_{0}$ is $95 \mathrm{~m}$ and that from $R$ to $Y_{0}$ is $155 \mathrm{~m}$. The density of simultaneously activated vehicles on each road is set to be the same, that is, $\lambda_{X_{i}}=\lambda_{Y_{j}}, \forall i, i \in \mathbb{Z}$, and is denoted by $\lambda_{V}$. Unless otherwise specified, the rest of network parameters are listed in the 
TABLE 1: Simulation parameters.

\begin{tabular}{lc}
\hline Parameter & Value \\
\hline Path loss exponents for I2V links and interference links $\left(\alpha_{S R}, \alpha_{S D}, \alpha_{I}\right)$ & 4 \\
Path loss exponent for the V2V R-D link $\left(\alpha_{R D}\right)$ & 2 \\
Infrastructure transmit power $\left(P_{S}\right)$ & $37 \mathrm{dBm}$ \\
Vehicle transmit power $\left(P_{V}\right)$ & $20 \mathrm{dBm}$ \\
Density of simultaneously activated infrastructures $\left(\lambda_{S}\right)$ & $10^{-7} \mathrm{~m}^{-2}$ \\
Density of simultaneously activated vehicles $\left(\lambda_{V}\right)$ & $10^{-3}$ vehicles $/ \mathrm{m}^{2}$ \\
Noise power $\left(\sigma^{2}\right)$ & $-99 \mathrm{dBm}$ \\
Distance between horizontal roads $\left(d_{\text {road, } X}\right)$ & $430 \mathrm{~m}$ \\
Distance between vertical roads $\left(d_{\text {road, } Y}\right)$ & $250 \mathrm{~m}$ \\
\hline
\end{tabular}

Table 1 , most of which are adopted from $[5,18,19]$. Certainly, in other system setups, the analytical method provided in Section 3 is also applicable.

Figure 3 displays the successful transmission probability achieved by the three schemes, when the average transmission rate $R_{0}$ changes. To verify the accuracy of the derivations of $P_{\mathrm{NC}}$ and $P_{\mathrm{DT}}$ presented in Section 3, we also carried out a Monte Carlo simulation (with 100,000 experiments) to find these values. It can be clearly seen that the analytical and simulation results are in line with each other. In addition, we can observe that, for a wide range of the transmission data rate, the NC scheme performs better than the DT and $\mathrm{RC}$ schemes. When the number of messages $M$ increases, the successful transmission probabilities of the three schemes all reduce, since now it is easier for $D$ to make mistakes in the source message recovery process. In this case, the performance gain of the NC scheme still remains, and a properly designed system should reduce the transmission data rate to improve the $\mathrm{I} 2 \mathrm{~V}$ communication reliability. Furthermore, we can also observe that if $R_{0}$ is chosen to be very large, the DT scheme would outperform the other two. This is because, for large transmission rate, under the current setup of transmit powers $P_{S}$ and $P_{V}$, the system is actually operating in a relatively low SINR regime. Since the NC scheme uses more channel resources to complete transmission, its information rate of each message is larger than that of the DT scheme. The decoding error probability at $R$ and $D$ is dominated by that of decoding high-rate messages. The situation is clearly more severe for the RC scheme. We can see that the successful transmission probability is too low to be acceptable in practice. Hence, again, a better system design should use a smaller rate (conveyed by each message) to deliver information. In this case, the system is operated in a relatively large SINR regime. The diversity and network coding gains of the NC scheme can be more beneficial, especially when a very high transmission reliability is demanded.

Figure 4 shows the successful transmission probability versus the vehicle density $\lambda_{V}$. It can be seen that for a wide range of interfering vehicle density, the NC scheme performs better than the DT scheme (the RC scheme is not shown due to its inferior performance). When the value of $\lambda_{V}$ is large, the impact of interference becomes large. The considered $\mathrm{I} 2 \mathrm{~V}$ communication system is operated in a low SINR regime. Again, due to the fact that the NC scheme

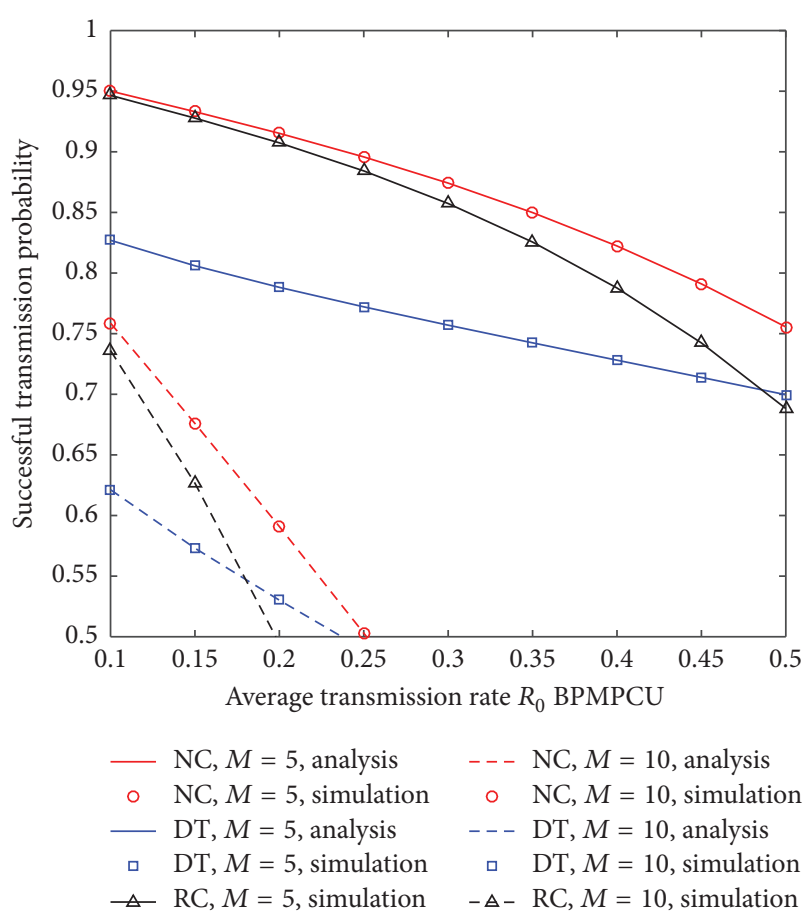

FIGURE 3: Successful transmission probability versus average transmission rate $R_{0}$. For the NC scheme, $L=3$.

demands each message to have a larger information rate, it may cause higher probability of incorrect decoding than the DT scheme. But since the successful transmission probability is too low, a lower transmission data rate $R_{0}$ should be chosen to guarantee a sufficiently high probability of successful transmission, when the density of simultaneously activated vehicles is large. It can be straightforwardly conjectured that the same observation would be made when the density of interfering infrastructures $\lambda_{S}$ is varying.

Finally, in Figure 5, we show the impact of changing the value of $L$ on the successful transmission probability. Note that when $L=0$, the NC scheme becomes the DT scheme. It can be directly seen that the NC scheme can perform better than the DT scheme. This is consistent with the previous observations. Moreover, different choices of $L$ would lead to different performance. But increasing $L$ 


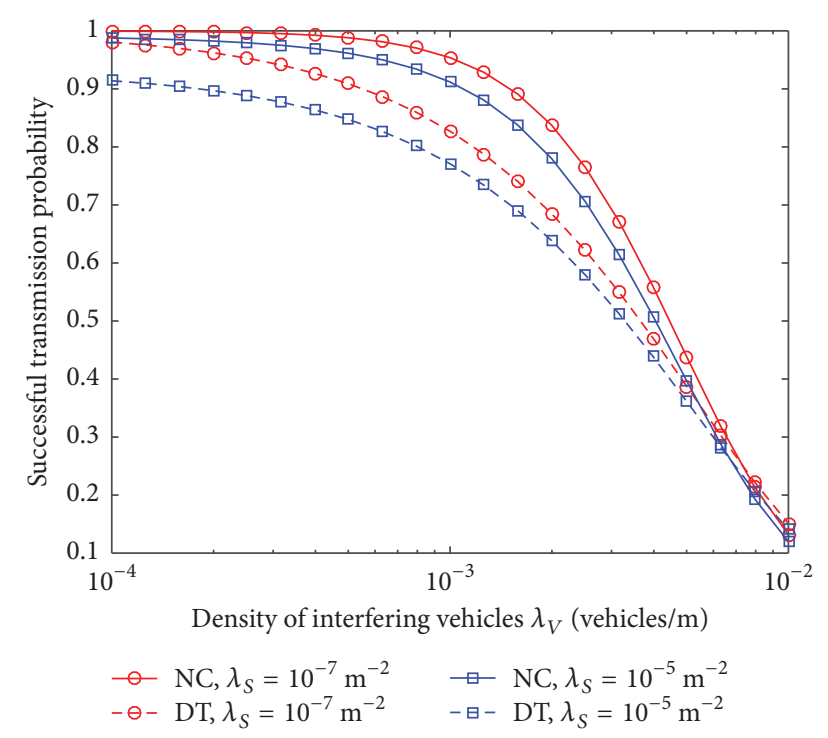

FIgURE 4: Successful transmission probability versus interfering vehicle density $\lambda_{V}$, with $M=5$ and $R_{0}=0.1 \mathrm{BPMPCU}$. For the NC scheme, $L=2$.

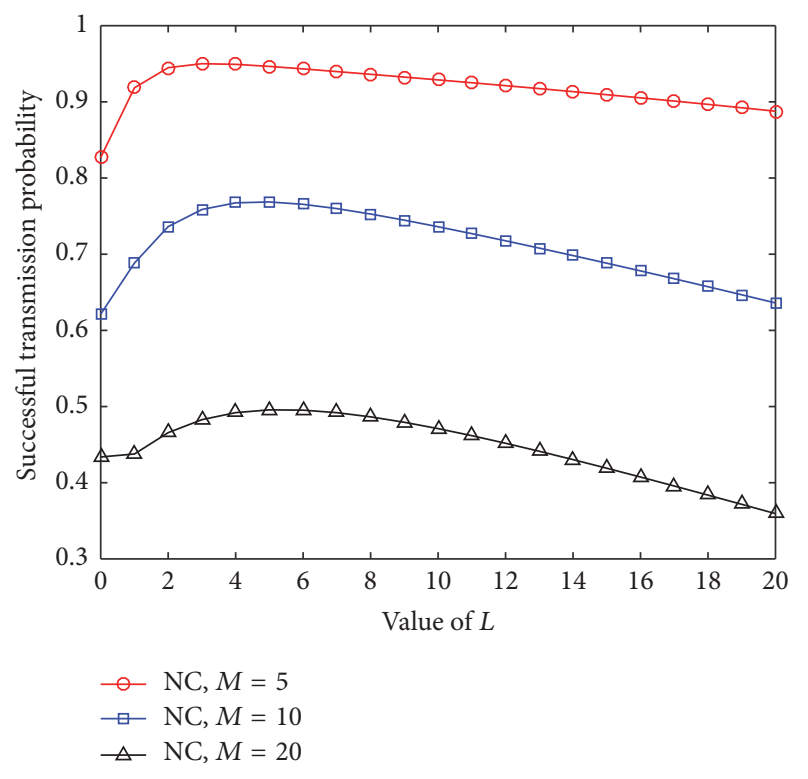

FIGURE 5: Successful transmission probability versus $L$ with $R_{0}=0.1$ BPMPCU.

does not necessarily improve the successful transmission probability. This is because although increasing $L$ provides the I2 $\mathrm{V}$ communication with higher diversity gain and power gain, the information rate of each message has to be increased to maintain a fixed average transmission rate. This may lead to difficulties in the decoding process and hence offset the benefits provided by the relay. It should be noted that the best choice of $L$ can vary significantly in different system setups. For instance, we can see from Figure 5 that different values of $M$ would require different choices of $L$ to achieve the best performance. In fact, the system parameters such as $M, P_{V}$, $P_{S}, \lambda_{S}$, and $\lambda_{V}$ would all affect the selection of $L$. In different systems, the analytical method presented in the above section can be applied to carry out the selection.

\section{Conclusion}

A typical communication scenario in ITS applications is the information distribution from roadside infrastructures to the vehicles within their coverage areas. To combat the negative impact of fading and cochannel interference, in recent years, investigations have proposed to use selected vehicles to serve as relays for such $\mathrm{I} 2 \mathrm{~V}$ transmission processes. In this paper, we have studied applying a network-coded cooperation strategy in an I2V communication system with multiple source messages. The potential cochannel interference generators are modeled based on stochastic geometry. Specifically, the interfering infrastructures are modeled by a 2D PPP and the interfering vehicles are modeled by $1 \mathrm{D} \mathrm{H}$-PPPs. Through deriving the successful transmission probability between an infrastructure and a vehicle, we have shown that the proposed transmission scheme can significantly improve the performance of the conventional direct I2V transmissions.

\section{Conflicts of Interest}

The authors declare that there are no conflicts of interest regarding the publication of this paper.

\section{Acknowledgments}

This work was supported in part by the National Natural Science Foundation of China (61331009, and 61401314), the Key Project of Science and Technology of Shanghai (Grant No. 15DZ1100801), the EU FP7 QUICK Project (PIRSES-GA2013-612652), and the Key Laboratory of System Control and Information Processing, Ministry of Education, Shanghai.

\section{References}

[1] K. Zheng, Q. Zheng, P. Chatzimisios, W. Xiang, and Y. Zhou, "Heterogeneous vehicular networking: a survey on architecture, challenges, and solutions," IEEE Communications Surveys and Tutorials, vol. 17, no. 4, pp. 2377-2396, 2015.

[2] D. Jiang and L. Delgrossi, "IEEE 802.11p: towards an international standard for wireless access in vehicular environments," in Proceedings of the 67th IEEE Vehicular Technology Conference (VTC '08), pp. 2036-2040, May 2008.

[3] B. Sikdar, "Comparison of broadcasting schemes for infrastructure to vehicular communications," IEEE Transactions on Intelligent Transportation Systems, vol. 13, no. 2, pp. 492-502, 2012.

[4] G. Araniti, C. Campolo, M. Condoluci, A. Iera, and A. Molinaro, "LTE for vehicular networking: a survey," IEEE Communications Magazine, vol. 51, no. 5, pp. 148-157, 2013.

[5] 3GPP TR 36.885 ver.14.0.0, "Study on LTE-based V2X Services," June 2016.

[6] A. Khezrian, T. D. Todd, G. Karakostas, and M. Azimifar, "Energy-efficient scheduling in green vehicular infrastructure with multiple roadside units," IEEE Transactions on Vehicular Technology, vol. 64, no. 5, pp. 1942-1957, 2015.

[7] A. A. Hammad, T. D. Todd, and G. Karakostas, "Variable-BitRate Transmission Schedule Generation in Green Vehicular 
Roadside Units," IEEE Transactions on Vehicular Technology, vol. 65, no. 3, pp. 1590-1604, 2016.

[8] M. J. Khabbaz, H. M. K. Alazemi, and C. M. Assi, "Modeling and delay analysis of a retransmission-based bundle delivery scheme for intermittent roadside communication networks," IEEE Transactions on Intelligent Transportation Systems, vol. 14, no. 2, pp. 700-708, 2013.

[9] A. Abdrabou and W. Zhuang, "Probabilistic delay control and road side unit placement for vehicular ad hoc networks with disrupted connectivity," IEEE Journal on Selected Areas in Communications, vol. 29, no. 1, pp. 129-139, 2011.

[10] T. Kimura, H. Saito, H. Honda, and R. Kawahara, "Modeling Urban ITS Communication via Stochastic Geometry Approach," in Proceedings of the 84th Vehicular Technology Conference (VTC-Fall) (IEEE'16), pp. 18-21, Montreal, QC, Canada, 2016.

[11] X. Ge, H. Cheng, G. Mao, Y. Yang, and S. Tu, "Vehicular Communications for $5 \mathrm{G}$ Cooperative Small-Cell Networks," IEEE Transactions on Vehicular Technology, vol. 65, no. 10, pp. 78827894, 2016.

[12] C. Yang, Y. Fu, Y. Zhang, S. Xie, and R. Yu, "Energy-efficient hybrid spectrum access scheme in cognitive vehicular Ad hoc networks," IEEE Communications Letters, vol. 17, no. 2, pp. 329332, 2013.

[13] T.-D. Nguyen, O. Berder, and O. Sentieys, "Energy-efficient cooperative techniques for infrastructure-to-vehicle communications," IEEE Transactions on Intelligent Transportation Systems, vol. 12, no. 3, pp. 659-668, 2011.

[14] R. Atallah, M. Khabbaz, and C. Assi, "Multihop V2I Communications: A Feasibility Study, Modeling, and Performance Analysis," IEEE Transactions on Vehicular Technology, vol. 66, no. 3, pp. 2801-2810, 2017.

[15] H. Topakkaya and Z. Wang, "Wireless network code design and performance analysis using diversity-multiplexing tradeoff," IEEE Transactions on Communications, vol. 59, no. 2, pp. 488496, 2011.

[16] R. W. Yeung, S.-Y. Li, N. Cai, and Z. Zhang, Network Coding Theory, NOW publishers, Inc, Breda, Netherlands, 2006.

[17] M. Xiao, J. Kliewer, and M. Skoglund, "Design of network codes for multiple-user multiple-relay wireless networks," IEEE Transactions on Communications, vol. 60, no. 12, pp. 3755-3766, 2012.

[18] N. Lu, N. Zhang, N. Cheng, X. Shen, J. W. Mark, and F. Bai, "Vehicles meet infrastructure: toward capacity-cost tradeoffs for vehicular access networks," IEEE Transactions on Intelligent Transportation Systems, vol. 14, no. 3, pp. 1266-1277, 2013.

[19] Z. Tong, H. Lu, M. Haenggi, and C. Poellabauer, "A Stochastic Geometry Approach to the Modeling of DSRC for Vehicular Safety Communication," IEEE Transactions on Intelligent Transportation Systems, vol. 17, no. 5, pp. 1448-1458, 2016.

[20] M. Haenggi, Stochastic Geometry for Wireless Networks, Cambridge University Press, Cambridge, England, UK, 2012.

[21] M. Haenggi and R. K. Ganti, "Interference in Large Wireless Networks," Foundations and Trends in Networking, vol. 3, no. 2, pp. 127-248, 2009. 


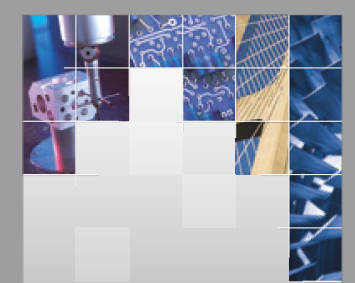

\section{Enfincering}
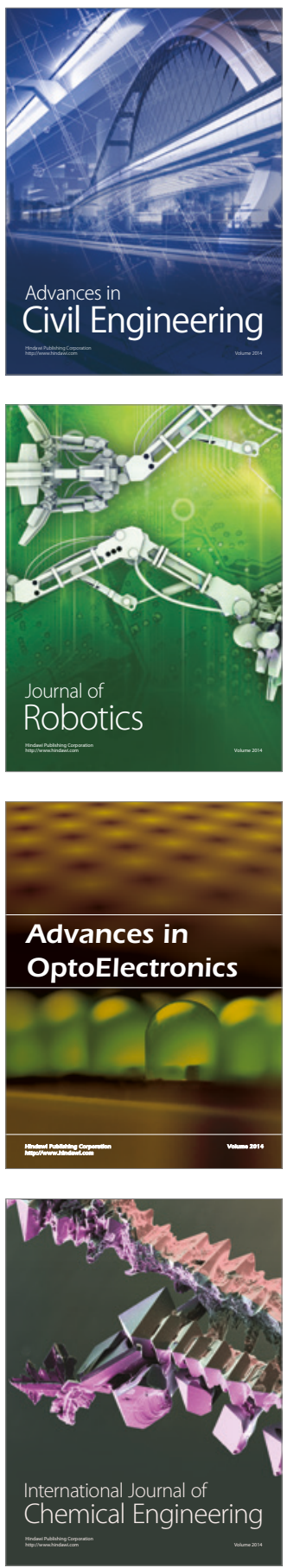

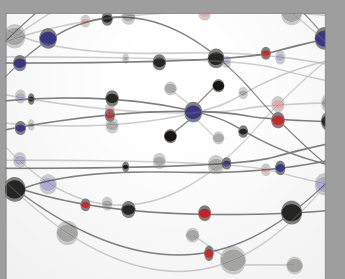

The Scientific World Journal

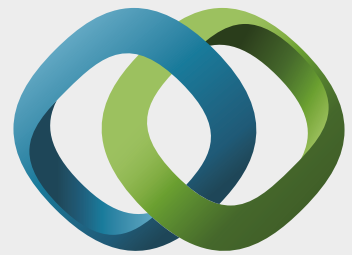

\section{Hindawi}

Submit your manuscripts at

https://www.hindawi.com
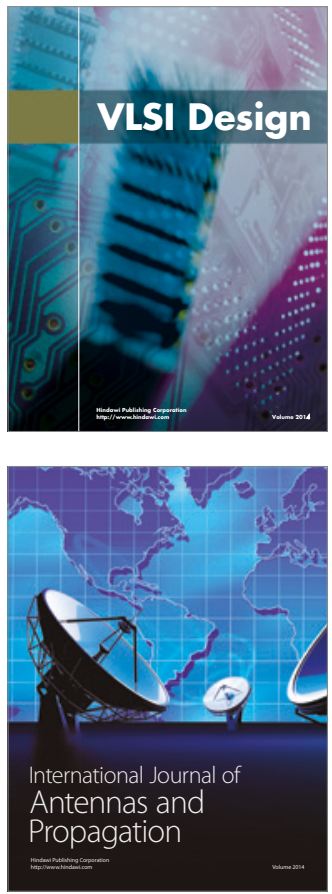

\section{Rotating}

Machinery
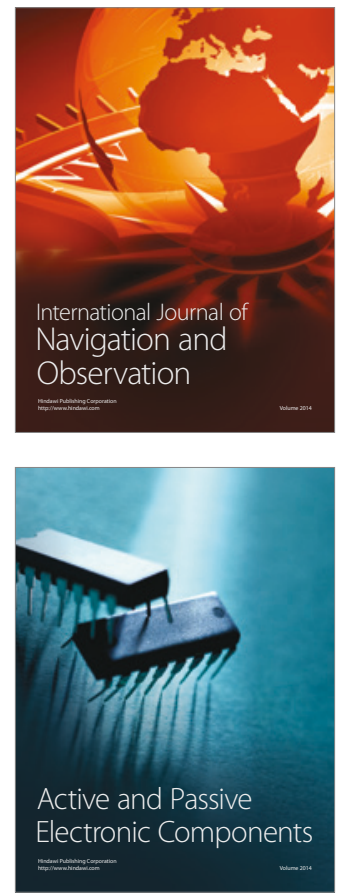
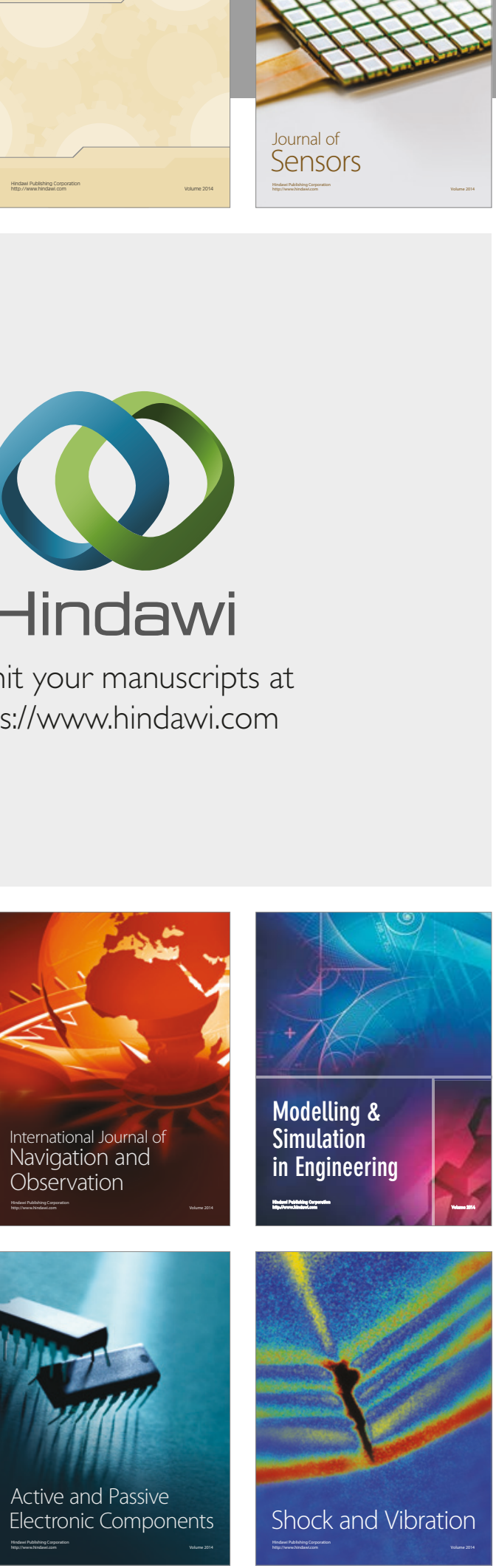
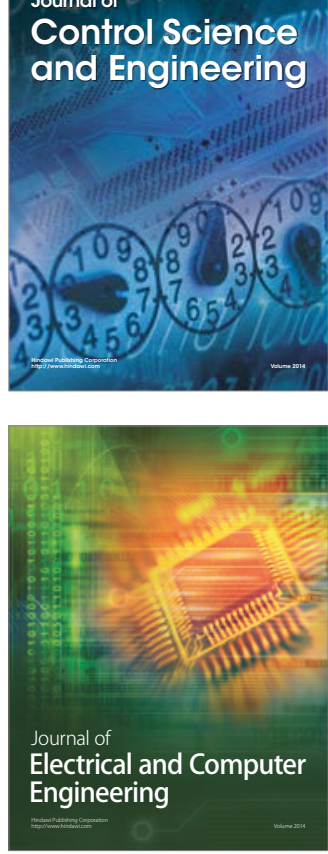

Distributed

Journal of

Control Science

and Engineering
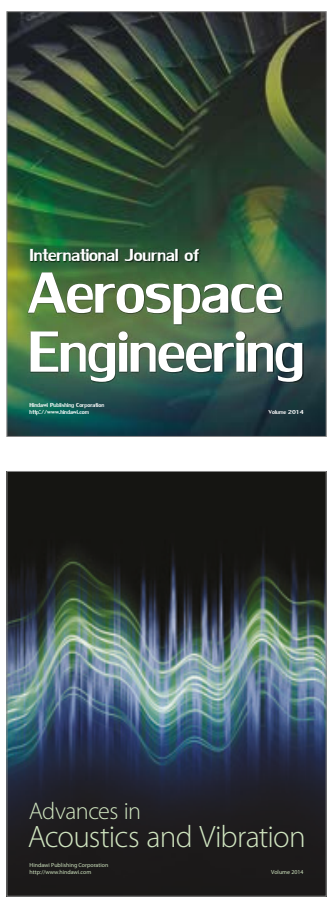

Sensor Networks 